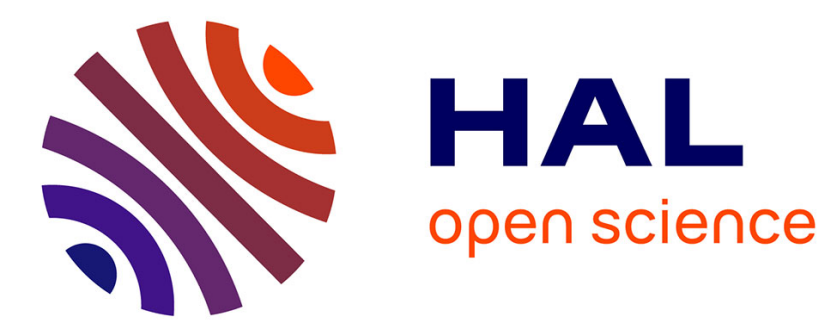

\title{
Scale by scale anisotropy in freely decaying rotating turbulence
}

\author{
Alexandre Delache, Claude Cambon, Fabien S. Godeferd
}

\section{To cite this version:}

Alexandre Delache, Claude Cambon, Fabien S. Godeferd. Scale by scale anisotropy in freely decaying rotating turbulence. Physics of Fluids, 2014, 26, 025104 (19 p.). 10.1063/1.4864099 . hal-01296835

\section{HAL Id: hal-01296835 \\ https://hal.science/hal-01296835}

Submitted on 8 Apr 2016

HAL is a multi-disciplinary open access archive for the deposit and dissemination of scientific research documents, whether they are published or not. The documents may come from teaching and research institutions in France or abroad, or from public or private research centers.
L'archive ouverte pluridisciplinaire HAL, est destinée au dépôt et à la diffusion de documents scientifiques de niveau recherche, publiés ou non, émanant des établissements d'enseignement et de recherche français ou étrangers, des laboratoires publics ou privés. 


\title{
Scale by scale anisotropy in freely decaying rotating turbulence
}

\author{
Alexandre Delache, ${ }^{1, a)}$ Claude Cambon, ${ }^{2, b)}$ and Fabien Godeferd ${ }^{2, c)}$ \\ ${ }^{1}$ LMFA UMR 5509 CNRS - site de Saint-Étienne, Université de Lyon, Université Jean \\ Monnet de Saint-Étienne, 42023 Saint-Etienne, France \\ ${ }^{2}$ LMFA UMR 5509 CNRS - École centrale de Lyon, Université de Lyon, 69134 Ecully, France
}

(Received 24 July 2013; accepted 22 January 2014; published online 10 February 2014)

\begin{abstract}
We analyse the anisotropy present at different scales in rotating homogeneous turbulence. The Zeman wavenumber $k_{\Omega}$ [O. Zeman, "A note on the spectra and decay of rotating homogeneous turbulence," Phys. Fluids 6, 3221 (1994)] was introduced to quantify the effect of nonlinearity compared to the Coriolis force, and was proposed as a threshold to separate the anisotropic subrange, at scales $k \ll k_{\Omega}$ (largest scales), from the isotropic subrange at $k \gg k_{\Omega}$. We study the nature of anisotropy and the role of the Zeman wavenumber using high resolution direct numerical simulations of freely decaying rotating turbulence, for various initial Rossby numbers. The anisotropy is analysed scale-by-scale by considering the angle-dependent energy spectrum, which characterizes directional anisotropy as a tendency to selectively accumulate energy towards the transverse waveplane $k_{\|}=0$. This ring-to-ring anisotropy is shown to develop in a non-monotonic way from small to large wavenumbers, first increasing, then decreasing. The Zeman wavenumber appears to be a suitable scale for estimating the separation between anisotropic and isotropic ranges. Consistently, when $k_{\Omega}$ is larger than the dissipative wavenumber cut-off, even the smallest scales are significantly anisotropic. This situation occurs eventually in our simulations of freely decaying turbulence, and is also observed in recent experimental results. Our investigation of anisotropy is completed by analyzing the angle-dependent nonlinear energy transfer and separate poloidal and toroidal energy spectra. The excess of poloidal energy in the equatorial plane $k_{\|}=0$ is linked with more energetic vertical motion. ( 2014 AIP Publishing LLC. [http://dx.doi.org/10.1063/1.4864099]
\end{abstract}

\section{INTRODUCTION}

In astrophysical, geophysical, and engineering flows, turbulence can be strongly affected by solid-body rotation, which often interacts with other effects arising from the presence of density variations or of solid boundaries. Here, we consider the idealized case of unbounded rotating turbulence in absence of additional distortion or forcing.

From several previous studies of this academic case, it appears that anisotropy is an essential feature of rotating flows, although it can be very different from turbulent shear flows anisotropy, which is due to anisotropic production by mean velocity gradients. The mechanism of anisotropy creation in rotating turbulence is more subtle, and has been discussed by several authors, ${ }^{1-3}$ with sometimes diverging viewpoints.

We focus in the present work on the question as to whether the anisotropy of rotating turbulence is present at all scales, and on means of its characterization. A first simple dimensional analysis suggests to delineate the range of anisotropic scales using a single threshold wavenumber based on

\footnotetext{
a)Electronic mail: alexandre.delache@univ-st-etienne.fr

b)Electronic mail: claude.cambon@ec-lyon.fr

c) Electronic mail: fabien.godeferd@ec-lyon.fr 
system vorticity $2 \Omega$ and on kinetic energy dissipation rate $\varepsilon$ :

$$
k_{\Omega}=\left(\frac{\Omega^{3}}{\varepsilon}\right)^{1 / 2}
$$

as proposed by Zeman. ${ }^{4}$ The corresponding threshold length scale would then be $\sim k_{\Omega}^{-1}$. Similar proposals can be made for shear flows ${ }^{5}$ and for stably stratified flows. ${ }^{6}$

On the one hand, this dimensional analysis suggests that isotropy in rotating turbulence is restored for wavenumbers larger than $k_{\Omega}$. On the other hand, there is no obvious supporting phenomenology for this separation, because of the peculiar features of unforced rotating turbulence: no direct production of energy by the Coriolis force; poor relevance of conventional anisotropy indicators such as one-point statistics - e.g., the deviatoric part of the Reynolds stress tensor - very specific role of the anisotropic dispersive inertial waves. ${ }^{7}$ We recall that the introduction by Zeman ${ }^{4}$ of a threshold wavenumber $k_{\Omega}$ - or similarly of a lengthscale in physical space - concerned a particular context: even if decaying rotating turbulence was addressed, some scaling equations came from the study of turbulence in the core of trailing vortices (contrails), with an adapted Lagrangian approach in the downstream direction. This led to a Zeman cut-off wavenumber based on $\Omega$ instead of $2 \Omega$ in Eq. (1), but later usage varies among authors, ${ }^{3,8}$ and we choose the first option, in absence of further decisive criterion.

The relevance of $k_{\Omega}$ seems to be ascertained in recent forced Direct Numerical Simulations (DNS) of rotating turbulence of Mininni et al.: ${ }^{8}$ isotropy is clearly shown to be restored for $k>k_{\Omega}$. This phenomenon is valid when $k_{\Omega}$ is in the inertial range, so the return to isotropy is not merely due to a viscous effect. A possible interference in these simulations is that anisotropy was the largest at the forcing (small) wavenumber, without possibility in such DNS to disentangle deterministic helical (ABC) forcing from anisotropic forcing.

Accordingly, and in spite of the advantage of forcing in terms of Reynolds number, we avoid the interference of artificial forcing anisotropy with the "natural" anisotropy, and we focus on high resolution DNS of decaying rotating turbulence, initiated with exactly isotropic initial data. A survey of a few previous experimental, numerical, and theoretical studies in this context is given as follows.

The experimental approach by Jacquin et al. ${ }^{9}$ showed that the anisotropy reflected in the Reynolds stress tensor is weak, in contrast with the anisotropy quantified by integral length scales with axial separation. This supports a detailed analysis of anisotropy for two-point statistics with spatial separation, ${ }^{10}$ or equivalently in Fourier space for all components and all wave vectors (see Sagaut and Cambon ${ }^{11}$ and references therein). In addition, both a macro-Rossby number and a micro-Rossby number are shown to be relevant, defined as

$$
R o^{L}=\frac{u^{\prime}}{2 \Omega L} \quad \text { and } \quad R o^{\omega}=\frac{\omega^{\prime}}{2 \Omega}
$$

respectively. $L$ is the integral length scale, $u^{\prime}$ the $r m s$ velocity, and $\omega^{\prime}$ the $r m s$ vorticity. The value $R O^{L}=1$ is obtained when the integral length scale matches the Zeman scale $l_{\Omega}=\left(\epsilon / \Omega^{3}\right)^{1 / 2}$ suggested by a balance between inertia and Coriolis force. Thus, the macro-Rossby number is also equivalent to $R o^{L}=\left(l_{\Omega} /(2 L)\right)^{2 / 3}$. The micro-Rossby number $R o^{\omega}$ was confirmed by Cambon et al. ${ }^{1}$ to characterize an additional structural transition related to polarization anisotropy (see below) when crossing the unit value. Smaller scales dynamics is compared to Coriolis effects in $R o^{\omega}$. Overall, both Rossby numbers in Eq. (2) help picture the action of rotation on large and small scales.

The role of the Zeman wavenumber, as a threshold above which small scale isotropy is restored, is not easy to understand because the anisotropy of rotating turbulence is both complex and subtle in freely decaying rotating turbulence with initial isotropic conditions. On the one hand, there is no simultaneous production of energy and of anisotropy at the largest scales, because the Coriolis force produces no energy, and the rise of anisotropy is a nonlinear effect, in contrast with a flow in the presence of a mean shear. 
We propose to characterize the anisotropy with spectra of two-point correlation functions, in Fourier space, with two objectives:

(1) To evaluate the anisotropic properties of the flow when separating axial and transverse contributions - of the wave vector for kinetic energy density spectrum, of the two-point separation vector for the second-order structure function - with respect to the rotation axis, and we also consider intermediate directions. Thus, the first relevant statistical quantity to be investigated is the angle-dependent energy spectrum. It is also the counterpart of the second-order structure function when considering velocity increments along different separation directions.

(2) To introduce a finer characterization of the structure of rotating turbulence via quantities that can be related to the directional variability of the flow for different velocity components, i.e., the axial/transverse gradients anisotropy. The relevant two-point quantity is the polarization anisotropy, which is easily computed only in Fourier space, as shown in Sec. III A.

Briefly speaking, the directional anisotropy of item 1 is identified on the angle-dependent energy spectrum. It reflects a trend towards two-dimensionalisation, with a pile-up of energy in the waveplane normal to the rotation axis, in the plane $k_{\|}=0$. The polarization anisotropy of item 2 tells how the energy is distributed in terms of poloidal and toroidal velocity components, for any direction of the wave vector. When restricted to the exact two-dimensional manifold $k_{\|}=0$, toroidal energy is associated with axial vortices and transverse velocity, or "vortical" structures, whereas poloidal energy is associated with axial velocity, or "jetal" structures. Both anisotropies are required to describe the quasi-two-dimensional three-component state (2D-3C), which is more complex than the classical 2D-2C state of purely horizontal 2D flow. A synthetic model is introduced for explaining this kind of anisotropy. One thus understands clearly that polarization anisotropy, as a difference of toroidal and poloidal contributions, is useful for refining the analysis of the structure of rotating turbulence.

The most recent very high resolution $\mathrm{DNS}^{8}$ confirm the role of the Zeman $k_{\Omega}$ wavenumber, but only a short Coriolis subrange appears with small scale separations and only one value is given to the rotation rate. Moreover, these are helically forced simulations, with a forcing term which is anisotropic at large scales and competes with the "natural" anisotropy created by Coriolis force at large scales. Therefore, to complete this analysis, we analyse results of new DNS of unforced rotating turbulence for four rotation rates, aiming at a resolution large enough to be able to locate clearly the Zeman wavenumber within the inertial range of turbulence, and obtain a separation of the large scales and dissipative ones.

Our numerical investigation is also very closely related to the recent Gyroflow experiments, ${ }^{10}$ in which a measure of the complete anisotropic energy transfer is done. Thanks to improvements of the experimental set-up to remove most of the global inertial modes in the tank, the experimental context can be considered to be very close to the numerical one. In addition, with refined particle image velocimetry (PIV) techniques, the authors can separate the Zeman and dissipative scales, although the latter cannot be resolved experimentally. In the following, we start by shortly presenting the equations and numerical technique in Sec. II. In Sec. III we discuss the results, considering the three aspects of anisotropy: directional (Sec. III A) and polarization (Sec. III D) for two-point velocity spectra, and the energy transfers (Sec. III E) as third-order statistics relevant for the dynamics. Conclusions are given in Sec. IV.

\section{GOVERNING EQUATION AND NUMERICAL SET-UP}

We consider the equation of fluid motion for the velocity field $\boldsymbol{u}$, i.e., the Navier-Stokes equation in the rotating frame:

$$
\left(\frac{\partial}{\partial t}-v \nabla^{2}\right) \boldsymbol{u}(\boldsymbol{x}, t)+\nabla p+(2 \boldsymbol{\Omega}+\boldsymbol{\omega}) \times \boldsymbol{u}=0,
$$

where $\omega=\nabla \times \boldsymbol{u}$ is the fluctuating vorticity, $p$ represents the total pressure modified by the centrifugal term, and divided by the mass density, and $v$ is the kinematic viscosity. The system rotation $\boldsymbol{\Omega}$ is imposed, without lack of generality, to be in the vertical direction: $\boldsymbol{\Omega}=(0,0, \Omega)$ with $\Omega$ the 
TABLE I. Recap of the parameters used in the simulations. Turbulence characteristics at the final time $t_{f}: u^{\prime} r m s$ of velocity, $L$ integral length scale, $\varepsilon$ dissipation, $\eta=\left(v^{3} / \varepsilon\right)^{1 / 4}$ Kolmogorov length scale, and $k_{\Omega}$ Zeman wavenumber. $N$ is the number of grid points in each direction of the Cartesian coordinate and in every simulation, the kinematic viscosity is $v=1 / 9000$, close to Kaneda's group data. ${ }^{14}$ The initial eddy turnover times are $\tau_{0} \simeq 0.76$ and $\tau_{0}^{\prime} \simeq 1.77$.

\begin{tabular}{lccccccccccc}
\hline \hline Run & $N$ & $t_{f}$ & $2 \Omega$ & $u^{\prime}$ & $L$ & $R e_{L}$ & $R o^{L}$ & $R o^{\omega}$ & $\varepsilon$ & $k_{\Omega}$ & $k_{\max } \eta$ \\
\hline$A_{0}$ & 1024 & $\tau_{0}$ & \multicolumn{1}{c}{0} & 0.38 & 0.46 & 1590.8 & $\infty$ & $\infty$ & 0.14 & 0 & 0.6 \\
$A_{1}$ & 1024 & $\tau_{0}$ & 12.57 & 0.41 & 0.52 & 1903.7 & 0.06 & 2.25 & 0.089 & 52.82 & 0.68 \\
$A_{2}$ & 1024 & $\tau_{0}$ & 6.28 & 0.39 & 0.52 & 1817.1 & 0.12 & 5.16 & 0.116 & 16.37 & 0.63 \\
$A_{3}$ & 1024 & $\tau_{0}$ & 3.14 & 0.39 & 0.49 & 1701.3 & 0.25 & 11.03 & 0.132 & 5.41 & 0.61 \\
$A_{4}$ & 1024 & $\tau_{0}$ & 1.57 & 0.38 & 0.47 & 1630.0 & 0.51 & 22.43 & 0.137 & 1.88 & 0.61 \\
$B_{1}$ & 2048 & $1.93 \tau_{0}^{\prime}$ & 21.7 & 0.53 & 1.10 & 5204.3 & 0.022 & 0.55 & 0.016 & 282.54 & 2.89 \\
$B_{2}$ & 2048 & $1.93 \tau_{0}^{\prime}$ & 5.42 & 0.46 & 1.15 & 4777.79 & 0.074 & 2.88 & 0.027 & 27.15 & 2.58 \\
\hline \hline
\end{tabular}

angular velocity. The fluid is considered to be incompressible, with divergence free velocity field $\nabla \cdot \boldsymbol{u}=0$.

Assuming periodic boundary conditions in the three directions of space, we solve numerically Eq. (3) by a pseudo-spectral method in a three-dimensional box of size $2 \pi$, using a parallel code and fast Fourier transforms ${ }^{12}$ with a resolution up to $2048^{3}$ collocation points. The pressure is solved by the Poisson equation obtained by taking the divergence of Eq. (3), consistently with the nondivergence of velocity. This is done algebraically in discretized Fourier space. Full dealiasing of the nonlinear term is done by spherical $2 / 3$ truncation rule ${ }^{13}$ for resolution $N^{3}=1024^{3}$ points, yielding a maximal resolved wavenumber $k_{\max }=341(=2 N / 3)$, or by the phase-shifting method ${ }^{13}$ at resolution $2048^{3}$, yielding $k_{\max }=965(=2 \sqrt{2} N / 3)$.

In addition to an isotropic simulation, we have performed simulations at six rotation rates, thus at different final Rossby numbers ranging from 0.02 to 0.5 , at the two different resolutions $1024^{3}$ and $2048^{3}$ points. A velocity field of fully developed homogeneous isotropic turbulence is used as initial conditions to the rotating runs, with two different choices:

- at $2048^{3}$ resolution, the velocity field comes from data from Kaneda's research group ${ }^{14}$ with the following characteristics: $u_{0}^{\prime} \simeq 0.57, L_{0} \simeq 1.01, \varepsilon_{0} \simeq 0.0832$, and eddy turnover time $\tau_{0}^{\prime} \simeq 1.77$

- at $1024^{3}$ resolution, the velocity field comes from our own simulation of homogeneous isotropic turbulence with $u_{0}^{\prime} \simeq 0.51, L_{0} \simeq 0.39, \varepsilon_{0} \simeq 0.35$, and eddy turnover time $\tau_{0} \simeq 0.76$;

where $u^{\prime}$ is the rms velocity, $\varepsilon=v\left\langle u_{i, j} u_{i, j}\right\rangle$ the dissipation rate. We define the integral length scale $L=\int_{0}^{\infty} R_{i i}(r) / R_{i i}(0) \mathrm{d} r$ where $R_{i j}(r)=\left\langle u_{i}(x) u_{j}(x+r)\right\rangle$ is the two-point velocity correlation tensor.

Table I provides the parameters at the last time $t_{f}$ of our simulations. In order to characterize the global evolution of turbulence, we provide the time evolution of Reynolds number $R e^{L}$ in Figure 1(a); of the integral length $L$ in Figure 1(d); of the macro Rossby number $R o^{L}$ in Figure 1(b); of the micro Rossby number $R o^{\omega}$ in Figure 1(c); of the dissipation in Figure 2(a); and of the Zeman scale $k_{\Omega}$ in Figure 2(b).

We observe that in freely decaying turbulence, the Reynolds number $R e^{L}$ is roughly constant after a short transient period. Accordingly, to compensate for the loss of energy, the integral length $L$ increases in time. The macro-Rossby number $R o^{L}$ and micro-Rossby number $R o^{\omega}$ have similar evolutions, although different magnitudes: they both decrease in time. The dissipation $\varepsilon$ also decreases in time, the consequence being that Zeman's scale $k_{\Omega}$ increases in time. Note that, between the different runs, increasing the rotation rate globally produces reduced dissipation $\varepsilon$ and larger Zeman's scale $k_{\Omega}$.

\section{ANISOTROPIC “2D-3C” STRUCTURE VERSUS UNIVERSAL 3D ISOTROPIC TURBULENCE}

We now present the modal decomposition of velocity which is easily done in spectral space, followed first by a discussion of the spherically averaged spectra, then by the analysis of the 

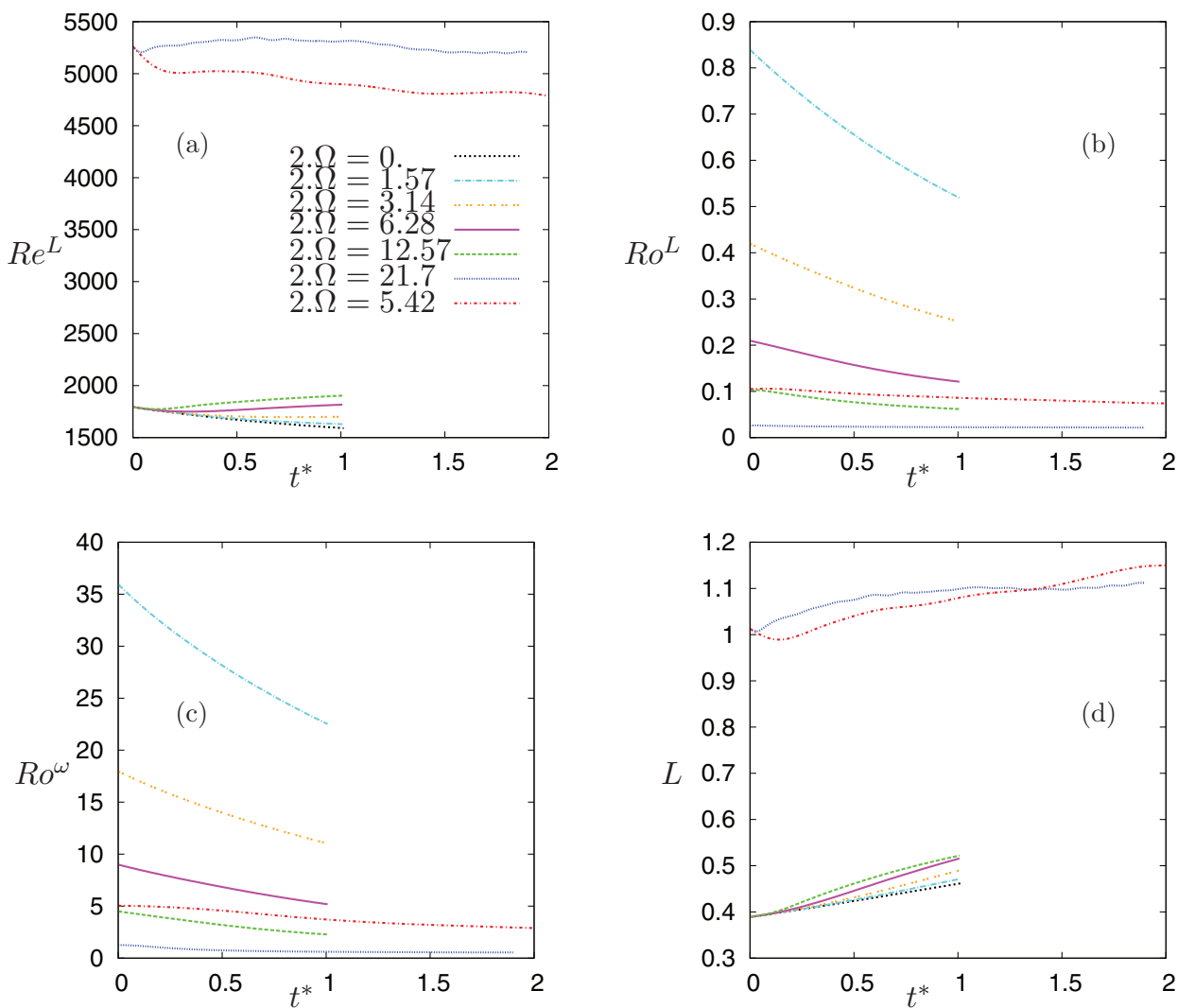

FIG. 1. For the different simulations, time evolution of: (a) Reynolds number $R e^{L}$ based on integral length $L$; (b) macroRossby number $R o^{L}$; (c) micro-Rossby number $R o^{\omega}$; and (d) integral length scale $L$. The non-dimensional time is $t^{*}=t / \tau_{0}$ where $\tau_{0}$ is the initial eddy time turnover. The legend of colored and dotted lines in (a), same for (b)-(d).
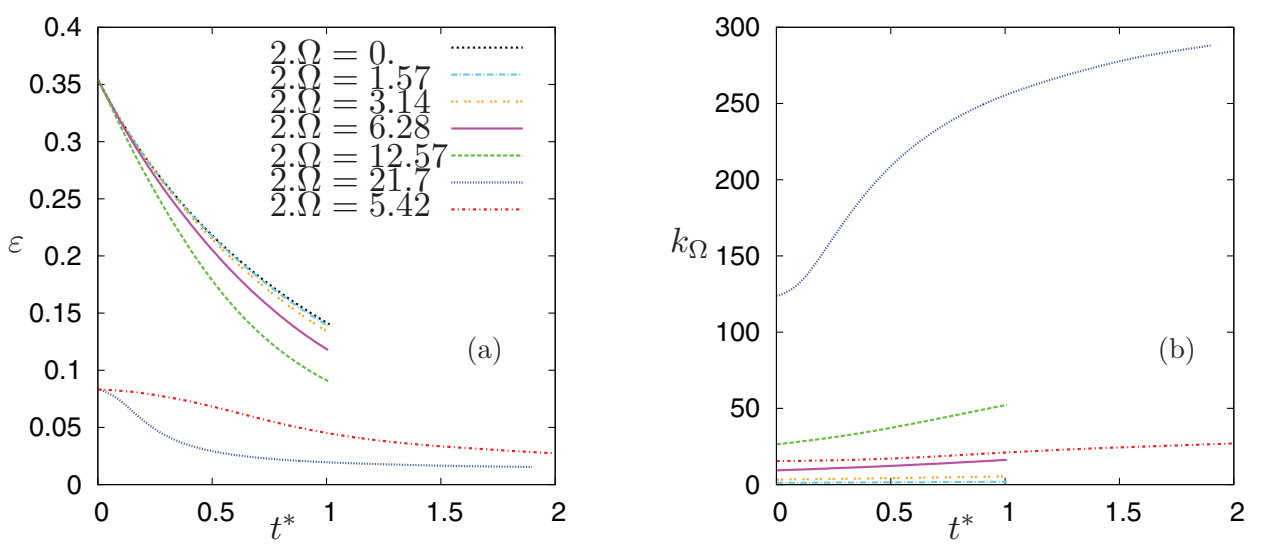

FIG. 2. For each simulation, time evolution of: (a) dissipation $\varepsilon$ and (b) Zeman scale $k_{\Omega}$. The legend of colored and dotted lines in (a), same for (b). 
direction-dependent spectra in the axisymmetric anisotropic description, finishing with the discussion of polarization anisotropy.

\section{A. Spectral equations and poloidal-toroidal decomposition}

From a theoretical viewpoint, it is important to note that the pseudo-spectral technique allows to obtain the fluctuating velocity field either in discretized physical space or in discretized Fourier space. Because neither the nonlinear term, here taken as the Lamb vector in Eq. (3), nor the Coriolis terms are divergence free, the gradient of pressure compensates to return the velocity field to solenoidal space. This corresponds to a simple geometrical projection when considering the 3D Fourier transform of Eq. (3):

$$
\left(\frac{\partial}{\partial t}+v k^{2}\right) \hat{\boldsymbol{u}}(\boldsymbol{k}, t)=-\mathbf{P}(\boldsymbol{k}) \cdot((2 \widehat{\boldsymbol{\Omega}+\boldsymbol{\omega})} \times \boldsymbol{u}) .
$$

The "overhat" denotes the 3D Fourier transformed spectral coefficient (possibly in the sense of distributions if the velocity field is a random variable). The solenoidal projection is ensured by the algebraic projector

$$
[\mathbf{P}(\boldsymbol{k})]_{i j}=\delta_{i j}-\frac{k_{i} k_{j}}{k^{2}}
$$

in which the second term $\boldsymbol{k} \otimes \boldsymbol{k} / k^{2}$ reflects contributions from pressure fluctuations. Accordingly, explicit pressure fluctuations are solved and removed from consideration, leaving linear and nonlinear contributions in the momentum equation:

$$
\left(\frac{\partial}{\partial t}+v k^{2}\right) \hat{\boldsymbol{u}}(\boldsymbol{k}, t)+\left(2 \Omega \frac{k_{\|}}{k}\right) \frac{\boldsymbol{k}}{k} \times \hat{\boldsymbol{u}}=-\mathbf{P}(\widehat{\boldsymbol{\omega} \times \boldsymbol{u}}) .
$$

Linear terms are gathered in the left-hand-side. The solenoidal projection of the Coriolis force exhibits system rotation modulated by an angle-dependent parameter $k_{\|} / k$, corresponding to the dispersion frequency of inertial waves $2 \Omega k_{\|} / k$. Note also that the Coriolis force disappears upon taking the inner product of Eq. (4) by the complex conjugate of the velocity in Fourier space $\hat{\boldsymbol{u}}^{*}$. This shows that the Coriolis force produces no energy at any scale.

Instead of using the solenoidal projector (5), one can use a two-component form of $\hat{\boldsymbol{u}}$ in order to satisfy the incompressibility condition, since the velocity vector $\hat{\boldsymbol{u}}(\boldsymbol{k})$ in Fourier space is perpendicular to $\boldsymbol{k}$ from the solenoidal property of the velocity vector in physical space: $\boldsymbol{\nabla} \cdot \boldsymbol{u}=$ $0 \Rightarrow \boldsymbol{k} \cdot \hat{\boldsymbol{u}}=0$. The three-component physical space velocity vector can therefore be represented by a two-component vector in the dual Fourier space and in the plane orthogonal to $k$ :

$$
\hat{\boldsymbol{u}}(\boldsymbol{k}, t)=u^{(1)}(\boldsymbol{k}, t) \boldsymbol{e}^{(1)}(\boldsymbol{k})+u^{(2)}(\boldsymbol{k}, t) \boldsymbol{e}^{(2)}(\boldsymbol{k}) .
$$

This simplification introduces the Craya frame of Refs. 11,15 shown in Figure 3. $u^{(1)}$ and $u^{(2)}$ are the toroidal and poloidal velocity components along the first two vectors of the zonal-meridional-radial orthonormal frame $\left(\boldsymbol{e}^{(1)}, \boldsymbol{e}^{(2)}, \boldsymbol{e}^{(3)}=\boldsymbol{k} / k\right)$ related to the system of polar-spherical coordinates in Figure 3.

Classically, to measure the energy scale by scale - or for each wave number $k$ in Fourier space - one uses averages of kinetic energy $E(k)$ over spheres $S_{k}$ of radius $k$ and thus averages out the anisotropic contents of the energy distribution:

$$
E(k, t)=\sum_{k \in S_{k}}|\hat{\boldsymbol{u}}(\boldsymbol{k}, t)|^{2} .
$$

In the discrete analysis of DNS data, the spherical shell is $S_{k}=\left\{k-\frac{\Delta k}{2}<|\boldsymbol{k}|<k+\frac{\Delta k}{2}\right\}$, where $\Delta k$ specifies the wavenumber increment in Fourier space. 

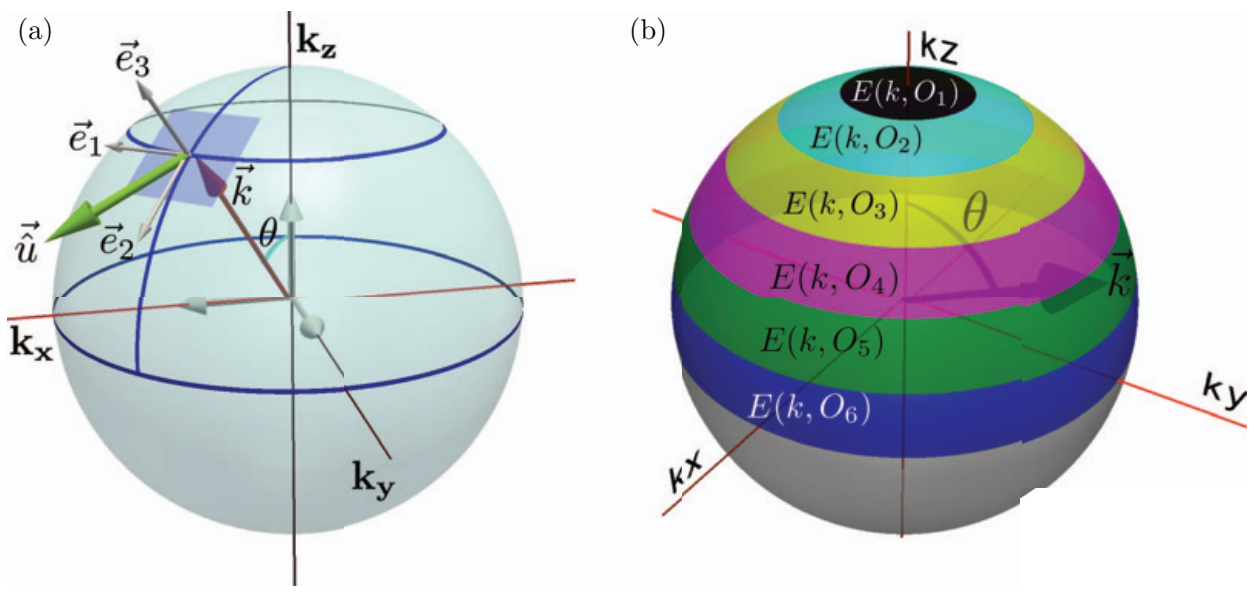

FIG. 3. (a) Craya frame and (b) diagram of angular spectral energy $E\left(k, O_{i}\right)$.

\section{B. Spherically averaged spectra}

In Figure 4, we plot the kinetic energy spectra $E\left(k, t=t_{f}\right)$ at final time $t_{f}$ for the different rotating cases, along with the initial spectrum for Figure 4(a), and with the initial and final spectra of the non-rotating case in Figure 4(b). We observe especially in Figure 4(a) with a large inertial range that, when the Rossby number decreases (higher rotation case), the energy spectrum is modified: the energy is higher at large scale (low wave number $k$ ) and smaller at small scale (high wave number $k$ ) exhibiting a modified dynamics of rotating turbulence. In the absence of energy production, the cascade is only quantified by a spectral energy transfer which drains energy from largest scales to smallest ones in order to feed the dissipation sink, hence a flux of energy strictly bounded by the amplitude of dissipation. Therefore, the strong reduction of the dissipation in the presence of rotation (see Table I) imposes a similar reduction of the interscale energy flux and therefore yields a steeper slope - less energy drain at small wavenumber, less energy sink at large wavenumber - with respect to the classical $k^{-5 / 3}$ Kolmogorov scaling of homogeneous isotropic turbulence, as also observed in other DNS. ${ }^{16}$ In recent experiments, the spectral slope is observed to be between -3 and $-2,{ }^{17}$ but it is computed from transverse velocity components so that the energy spectrum is given in terms of $k_{\perp}$, whereas our Figure 4 presents spherically averaged spectra $E(k)$. From phenomenological arguments, Zhou ${ }^{18}$ proposed the scaling $E \sim \sqrt{\Omega \varepsilon} k^{-2}$, slightly different from $E \sim k^{-11 / 5}$ proposed by Zeman. ${ }^{4}$ A theoretical approach of large Reynolds number, vanishing Rossby
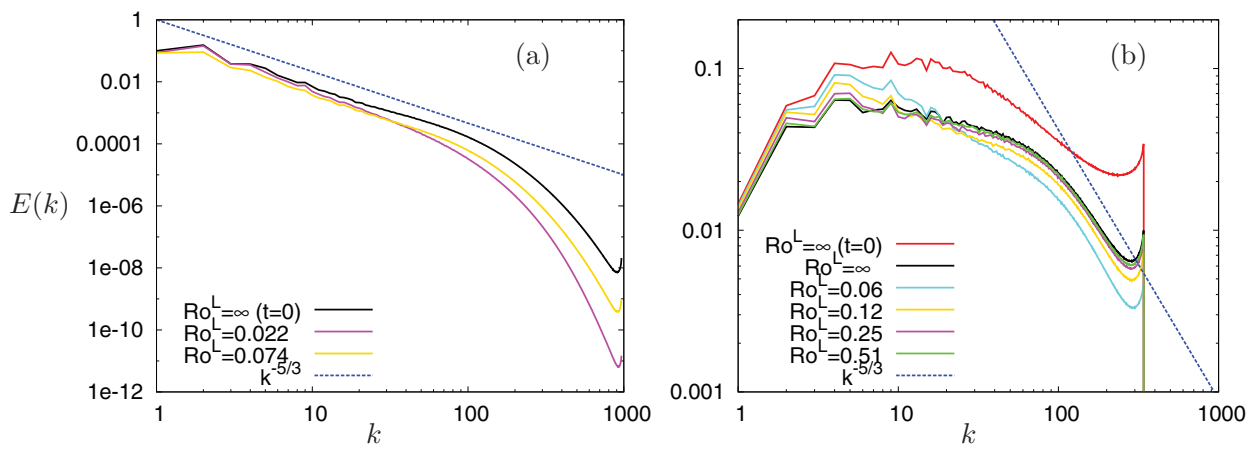

FIG. 4. Spectra of kinetic energy at $t=t_{f}$ for different Rossby numbers $R o^{L}$ and different resolutions: (a) $2048^{3}$ and (b) $1024^{3}$. 
number, turbulence, including full axisymmetric anisotropy, yields $E \sim k^{-3},{ }^{19}$ steeper than what we obtain in the current simulations. However, we observe no obvious trace of the Zeman wavenumber on the different kinetic energy spectra, for both resolutions at all rotating rates.

\section{Investigation of directional anisotropy}

In the case of rotating turbulence with axisymmetric statistics about the rotating axis, the distribution of energy is not equi-distributed over the spherical shell of radius $k$ in contrast to isotropic turbulence. We characterize this non-equi-distribution of kinetic energy by introducing the angular dependence of the kinetic energy spectrum $\mathcal{E}\left(k, O_{i}\right)$, as proposed in previous studies. ${ }^{11,20,21}$ In the discrete case, we decompose the sphere $S_{k}$ into several rings $O_{i}$ (six rings $i=1 \ldots 6$ in our simulations, as shown in Figure 3(b)):

$$
\mathcal{E}\left(k, O_{i}\right)=\frac{1}{m_{k}^{i}} \sum_{k \in O_{i}}\left(\left|u^{(1)}(\boldsymbol{k})\right|^{2}+\left|u^{(2)}(\boldsymbol{k})\right|^{2}\right)=\mathcal{E}^{(1)}\left(k, O_{i}\right)+\mathcal{E}^{(2)}\left(k, O_{i}\right),
$$

where $\mathcal{E}^{(1)}\left(k, O_{i}\right)$ and $\mathcal{E}^{(2)}\left(k, O_{i}\right)$ are the toroidal and poloidal contributions to $\mathcal{E}\left(k, O_{i}\right)$ which gives the shell-to-shell and ring-to-ring dependence of energy spectrum. The weight $m_{k}^{i}=$ $(\pi / 2)\left(2\left(\theta_{i}-\theta_{i+1}\right)\right)^{-1}\left(\left(\sin \left(\theta_{i}\right)-\sin \left(\theta_{i+1}\right)\right)^{-1}\right.$ is the normalisation term of the statistics, and the discretized angles are $\theta_{i}=(\pi / 2)((i-1) / 6)$. The choice of six rings $(i=1,2, \ldots, 6)$ to decompose the spherical shell $S_{k}=\bigcup_{i=1}^{6} O_{i}$ is a compromise between the number of shells and the number of rings in each shell to ensure sufficient statistical sampling within the rings. We consider only a half-shell because of the Hermitian symmetry $\hat{\boldsymbol{u}}(-\boldsymbol{k})=\hat{\boldsymbol{u}}^{*}(\boldsymbol{k})$. As a result, it is finally consistent to $E(k, t)=\sum_{i=1,6} m_{k}^{i} \mathcal{E}\left(k, O_{i}\right)$. Figures 5, 6(a), and 6(b) show the kinetic energy spectra at different angles $\mathcal{E}\left(k, O_{i}\right)$ for the different Rossby numbers $R^{L}$, therefore at different Zeman wavenumbers $k_{\Omega}$, and for the two resolutions. Enstrophy visualizations are shown in Figures 6(c) and 6(d). We notice that at high Rossby number (Figure 6(c)), the structures are close to the ones in isotropic turbulence whereas at low Rossby number (Figure 6(d)), they are axially oriented (we will rediscuss this visualization in Sec. III D 2).

In the isotropic case or at the largest Rossby number (low rotation rate case), we observe in Figures 5(a) and 5(b) that the angular spectra $E\left(k, O_{i}\right)$ in all the rings collapse at all scale, except at the largest ones, where statistical sampling is very coarse and explains the departure. Since the Zeman wavenumber $k_{\Omega}$ (shown by a vertical line in Figure $5(\mathrm{~b})$ ) is smaller than all the turbulent structures in the flow, rotation has no significant effect on the dynamics.

At increasingly low values of the Rossby number (higher rotation rate cases), in Figures 5(c), 5(d), and 6(a), an increasingly wide range of large scales exhibits a marked anisotropy in which energy concentrates on the equatorial ring $O_{6}$. The concerned range of wave numbers extends to the Zeman scale, i.e., for $k \leq k_{\Omega}$.

For wavenumbers above the Zeman wavenumber, i.e., $k \geq k_{\Omega}$, the spectra recover an isotropic distribution, such that all angle-dependent energy spectra collapse again. Below, that is at very large scales, isotropy seems to be partially restored as well, although statistical inaccuracies hide this in the $1024^{3}$ runs, but it seems to be the case in the $2048^{3}$ runs. Whether this is due to large-scale cut-off by the box size is yet to be determined. Finally, at very high rotation rate (very low Rossby number cases), in Figures 5(e) and 6(b), every scale exhibits anisotropic characteristics, down to the dissipative ones. Therefore, even at these quite large values of the Reynolds number, the turbulent structures may remain anisotropic, even the smallest ones, provided the Rossby number is small enough.

This concentration of energy on equatorial ring was already observed in DNS of rotating turbulence ${ }^{1}$ and in a statistical model of inertial wave turbulence. ${ }^{19}$ In all these results, the anisotropy is non-monotonic in terms of $k$. Its consistency with experimental results ${ }^{10,22}$ is discussed in Cambon et $\mathrm{al}^{23}$ In terms of spectral scaling, discussed above for $E(k)$, the wave turbulence model spectral scaling $E \sim k^{-3}$ comes from the average over angle-dependent spectra in which a $k^{-2}$ scaling is found for the most energetic spectrum. It corresponds to the equatorial $O_{6}$ spectrum in 

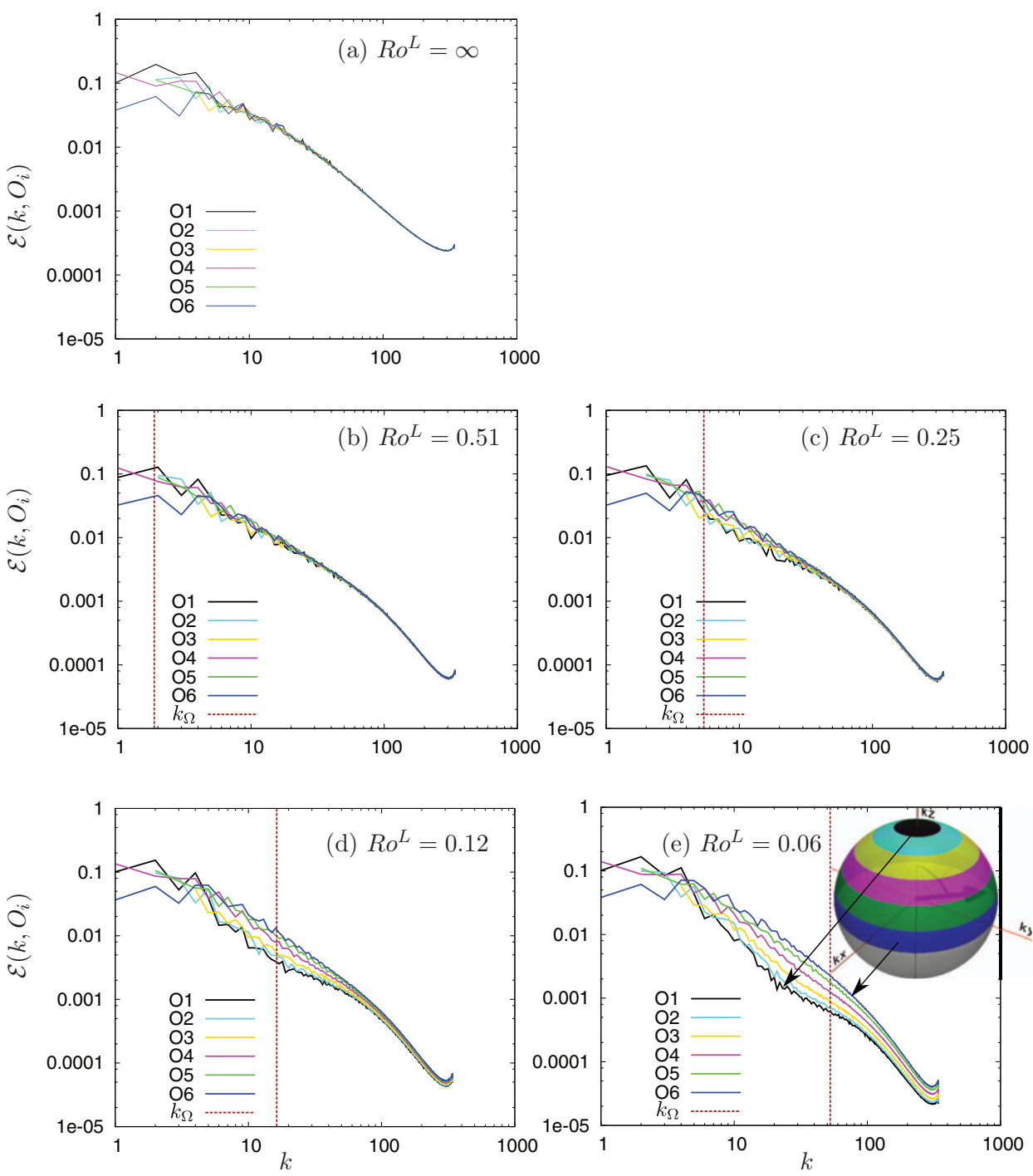

FIG. 5. Total angular spectral energy $\mathcal{E}\left(k, O_{i}\right)$ at $t=t_{f}$ for different Rossby numbers $R o^{L}$ and Zeman wavenumbers $k_{\Omega}$ (vertical lines) at resolution $1024^{3}$ : (a) Run $A_{0}, R o^{L}=\infty$; (b) $\operatorname{Run} A_{1}, R o^{L}=0.51$; (c) $\operatorname{Run} A_{2}, R o^{L}=0.25$; (d) $\operatorname{Run} A_{3}, R o^{L}$ $=0.12$; and (e) Run $A_{4}, R o^{L}=0.06$.

Figures 5 and 6. The $k^{-2}$ scaling is approximately recovered for the $O_{6}$ spectrum of the lower Rossby number simulation of Figure 6(b). We underline that the $k^{-3}$ classical scaling for $E(k)$ in 2D turbulence, in which the enstrophy is the relevant prefactor, is of a different nature from our spherically averaged angle-dependent.

The concentration in Fourier space of the energy towards the "equatorial ring" $O_{6}$ therefore reflects a trend towards two-dimensionalization, because $k_{\|} \simeq 0$ corresponds in physical space to structures with less variability in the axial direction. When time evolves, it means that the statistics corresponding to variability $\partial / \partial z$ decrease along the axis, which we call a quasi-2D tendency. Exact concentration on $k_{\|}=0$, as a Dirac distribution, is never achieved in previous studies, and would characterize Taylor columns. $^{24,25}$ 

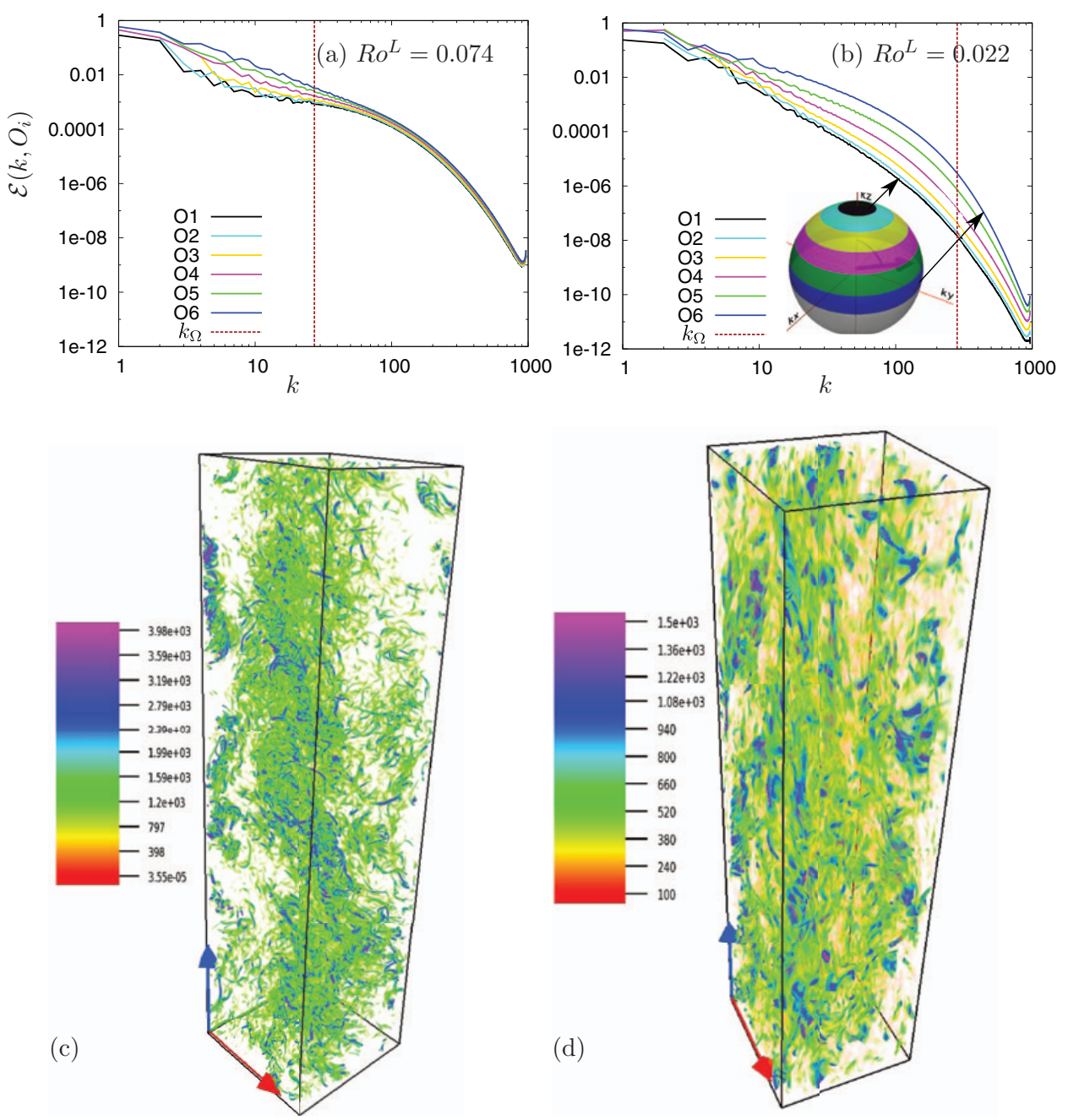

FIG. 6. Total angular spectral energy $\mathcal{E}\left(k, O_{i}\right)$ at $t=t_{f}$ for different Rossby number $R o^{L}$ at resolution $2048^{3}$ : (a) for Run $A_{5}$ at $R o^{L}=0.074$ and (b) for Run $A_{6}, R o^{L}=0.022$. Visualisation with vapor (thanks to NCAR software), of the enstrophy $\left\langle\omega^{2}\right\rangle$ distribution of the computational box (total vertical extent but only a $512^{2}$ subdomain horizontally is shown): (c) $R o^{L}$ $=0.074$ and $(\mathrm{d}) R o^{L}=0.022$.

As discussed hereinabove, the Zeman wavenumber $k_{\Omega}$ plays an important role for delineating the range of wavenumbers for which structures are closer to $2 \mathrm{D}$ from the range of wavenumbers over which the structures are mostly $3 \mathrm{D}$. For wavenumbers smaller than $k_{\Omega}$, the distribution of energy is strongly anisotropic and structured in agreement with the quasi-2D concept. The domain $\left[1, k_{\Omega}\right]$ is clearly a domain of increased anisotropy, domain which increases as $R o^{L}$ decreases, whereas the distribution of energy becomes again isotropic if $k$ is larger than $k_{\Omega}$. When $k_{\Omega}$ is larger than the largest wavenumbers in the flow, the structures at all scales may be significantly anisotropic.

In order to provide a quantitative scale-by-scale measure of the intensity of anisotropy, we introduce the anisotropy factor

$$
a(k)=\frac{\mathcal{E}\left(k, O_{6}\right)-\mathcal{E}\left(k, O_{1}\right)}{\mathcal{E}\left(k, O_{6}\right)+\mathcal{E}\left(k, O_{1}\right)}
$$


(a)

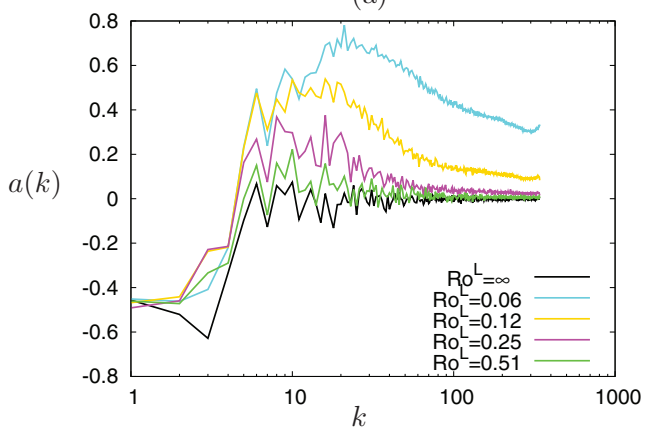

(b)

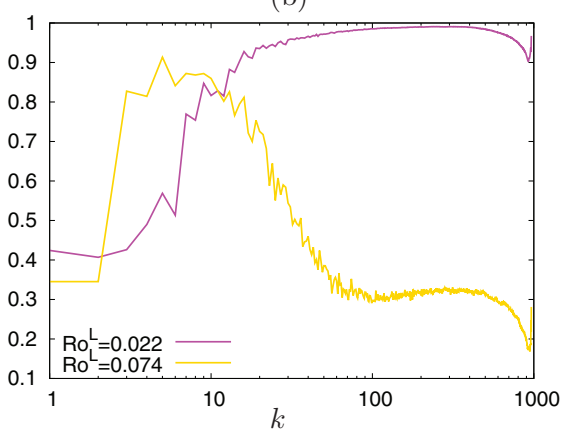

FIG. 7. Scale-by-scale anisotropy factor $a(k)$ for each Rossby number: (a) $1024^{3}$ DNS and (b) $2048^{3}$ DNS.

In Figure 7, we plot this factor for both resolutions. In the case of DNS with $1024^{3}$ points, Figure 7(a) shows that $a(k)<0$ for $k<\sim 5$, even in the isotropic case. This is due to a lack of statistical sampling in the largest scales, with only a few very large structures inherited from the initial conditions. When $k>5, a(k) \simeq 0$ in the isotropic case, logically, and the anisotropy factor becomes positive for the rotating cases. The maximum of anisotropy $a(k)$ appears at a given wave number $k_{\max }^{\text {aniso, }}$ that depends on the value of the Rossby number. For $2048^{3}$ DNS, Figure 7(b) shows that $a(k)>0$ at all scales, since sampling is much better in the large scales. This also means that energy is not equipartitioned, and is concentrated at the equator. The curve at $R o^{L}=0.074$ shows a strong, rather abrupt, reduction of $a(k)$ at small scales, although positive anisotropy is still present. The curve at $R O^{L}=0.022$ exhibits maximal anisotropy in a wide range of scales down to the smallest ones.

The wavenumber of maximum anisotropy $k_{\max }^{\text {aniso }}$ along with the Zeman wavenumber $k_{\Omega}$ are plotted against Rossby number in Figure 8 with a logarithmic scale. The figure shows a power-law scaling $k_{\Omega} \sim\left(R o^{L}\right)^{-1.6}$ for all simulations at both resolutions. $k_{\max }^{\text {aniso }}$ is however more sensitive to the resolution, and no particular scaling can be found from our few points on this graph. There does not

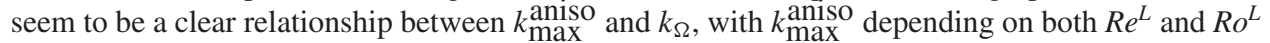
whereas $k_{\Omega}$ depends mostly on $R o^{L}$. In their experiments, Cortet and Moisy ${ }^{3}$ measured the two lengths equivalent to $k_{\Omega}$ and $k_{\max }^{\text {aniso }}$, namely, $l_{\Omega}$ and $r_{\text {aniso }}$, respectively, and found $r_{\text {aniso }} \simeq \max \left(l_{\Omega}, 2 \eta\right)$ where $\eta$ is the Kolmogorov scale. We do not observe this relationship in our simulations, but the experimental and numerical Rossby and Reynolds numbers are different.

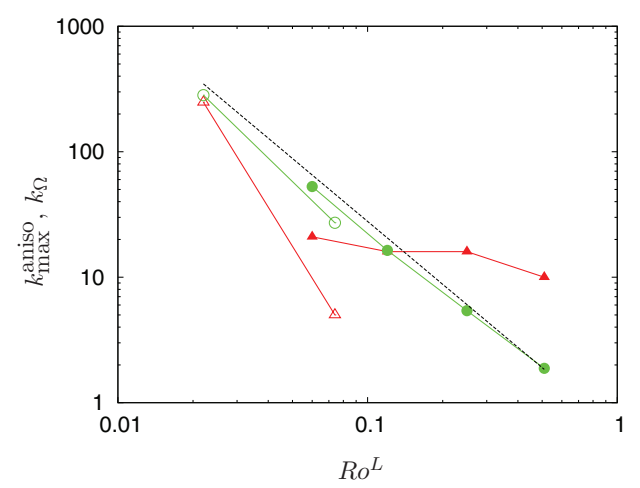

FIG. 8. For each simulation, we plot the following in terms of the Rossby number $R o^{L}$ : green circles: Zeman wavenumber $k_{\Omega}$; red triangles: wavenumber of maximum anisotropy in the angular spectra $k_{\max }^{\text {aniso }}$. Open symbols correspond to $2048^{3}$ simulations, filled symbols to $1024^{3}$ ones. A power-law $\left(R o^{L}\right)^{-1.6}$ is also proposed for the dependence of $k_{\Omega}$. 
In this work, we study the quasi 2D structure of the flow by examining its state at final time $t$ $=t_{f}$. However, we stress that it is the result of a dynamical phenomenon in freely decaying rotating turbulence started with initial isotropic turbulence. Indeed, when turbulence decays, the dissipation rate $\varepsilon$ decreases and the Rossby number $R o^{L}$ as well. A consequence, from its definition (1), is that the Zeman wavenumber $k_{\Omega}$ increases when $R o^{L}$ decreases and $\varepsilon$ decreases. This dependence raises two remarks:

- First, the anisotropic wavenumber range $\left[1, k_{\Omega}\right]$ correspondingly expands in time and eventually becomes the whole spectral domain. In the mean time, the anisotropy growth is expected to increase as $R o^{L}$ decreases. A means of compensating this would be to maintain a constant dissipation $\varepsilon$, or to force turbulence as done by Mininni et al. ${ }^{8}$ Nevertheless, the choice of a forcing method is in competition with the anisotropic characteristic of rotating turbulence whereas this anisotropic characteristic is free to develop in freely decaying turbulence. In both the forced or unforced cases and for all the initial conditions considered in the above-mentioned literature, the Zeman wavenumber appears to be a good indicator marking the scale at which isotropic distribution begins to be restored.

- Second, because $k_{\Omega}$ is defined in terms of $\varepsilon$ and $\Omega$, its scaling relies on the scaling of the dissipation rate, or more precisely on the scaling of a related lengthscale $L$. We recall the relationship with the Rossby number proposed in Sec. I: $R o^{L}=\left(l_{\Omega} /(2 L)\right)^{2 / 3}$ which suggests that $k_{\Omega}=1 / l_{\Omega} \sim\left(R o^{L}\right)^{-3 / 2} / L$. Since we observe in Figure 8 a rather clear powerlaw $k_{\Omega} \sim\left(R o^{L}\right)^{-1.6}$ which differs from -1.5 , we conclude that the length scale $L$ is altered by rotation and is thus also a function of the Rossby number.

\section{Investigating the polarization anisotropy}

\section{Definition and simple model for polarization anisotropy}

In Figures 5 and 6, we observed in Sec. III C that the kinetic energy is concentrated in the equatorial ring $O_{6}$ for wavenumbers smaller than the Zeman wavenumber $k_{\Omega}$, and tends to be equi-distributed for larger wavenumbers. This effect is enhanced when the Rossby number is small. In addition to this directional anisotropy quantified by energy difference between different rings, another anisotropy can be evidenced from the distribution of the velocity components within a given ring. This second kind of anisotropy is called polarization, by analogy with electromagnetic waves polarization, since they also involve an exactly transverse wave field with respect to the direction of the wave vector $\boldsymbol{k}$, as the spectral velocity vector $\hat{\boldsymbol{u}}$ is. The polarization anisotropy is precisely defined as the difference in statistics between the two components $u^{(1)}$ and $u^{(2)}$ at a given $\boldsymbol{k}$, in the plane normal to $\boldsymbol{k}$. If the energy is concentrated towards the equatorial ring — the quasi-2D state $u^{(1)}$ is close to a purely horizontal flow, and $u^{(2)}$ is close to a purely axial flow. The whole anisotropy therefore characterizes a trend towards a $2 \mathrm{D}-3 \mathrm{C}$ - two-dimensional, three-component - structure.

In order to quantify polarization anisotropy in our simulations, we have computed the toroidal $u^{(1)}$ and poloidal $u^{(2)}$ velocity components, and their respective angular distributed power spectra $\mathcal{E}^{(1)}\left(k, O_{i}\right)$ and $\mathcal{E}^{(2)}\left(k, O_{i}\right)$ defined in Eq. (9). The sum of the two yields the kinetic energy spectra. For a complete statistical spectral characterization, one would also require a cross-spectrum linked to $\left\langle u^{(2)} * u^{(1)}\right\rangle$, but this term is of negligible amplitude near the $2 \mathrm{D}$ manifold $k_{\|}=0$ in rotating turbulence. Dynamical equations can be obtained for the polarization spectrum, or for the toroidal and poloidal power spectra, ${ }^{1,11,20}$ briefly presented in the Appendix.

To illustrate the physical meaning of polarization, we introduce a polarized synthetic field, following Rogallo's method, ${ }^{26}$ but restricted to a strong directional anisotropy given by spectral contributions in the equatorial band $O_{6}$ and to low wave numbers such that $7 \leq k \leq 9$ (the wavevectors matching these conditions in a $256^{3}$ cartesian grid are used). The resulting incompressible velocity field model is

$$
\boldsymbol{v}(\boldsymbol{x})=\sum_{\boldsymbol{k} \in O_{6}, 7 \leq k \leq 9} \sqrt{e(k)} e^{\mathrm{i} \beta}\left[\cos (\alpha) \boldsymbol{e}^{(1)}(\boldsymbol{k})+\sin (\alpha) \boldsymbol{e}^{(2)}(\boldsymbol{k})\right] e^{\mathrm{i} \boldsymbol{k} \cdot \boldsymbol{x}}+\text { c.c. }
$$



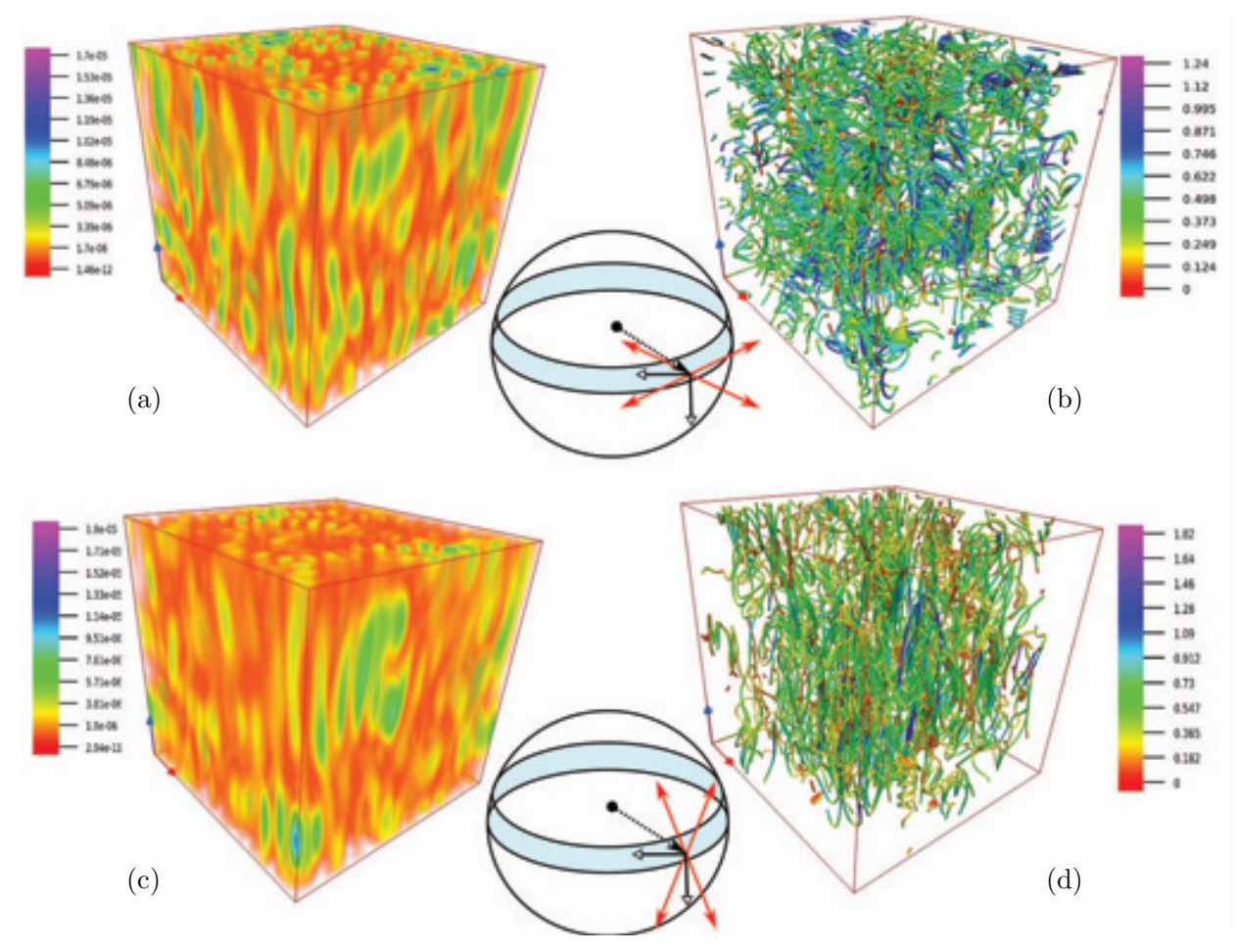

FIG. 9. Two "polarized" synthetic fields defined by Eq. (11), with the same energy accumulated in the equatorial spectral plane (shaded ring on the spheres that depict the same spectral sphere and coordinate system as in Fig. 3(a)) but with two different polarization ratios $\mathcal{E}^{(2)}\left(k, O_{i}\right) / \mathcal{E}^{(1)}\left(k, O_{i}\right)$, depicted by red arrows that show the possible corresponding orientations of the Fourier vectors $\hat{\boldsymbol{u}}$. Top figures (a) and (b): for $\mathcal{E}^{(2)}\left(k, O_{i}\right) / \mathcal{E}^{(1)}\left(k, O_{i}\right)=1 / 3$; bottom figures (c) and (d) for $\mathcal{E}^{(2)}\left(k, O_{i}\right) / \mathcal{E}^{(1)}\left(k, O_{i}\right)=3$. (a) and (c) The vorticity distribution; (b) and (d) 300 randomly picked streamlines.

where c.c. is complex conjugate, $e(k) \simeq 0.2$ is the energy of each wavenumber, $\beta$ is a random phase uniformly distributed in $[0,2 \pi]$ (at each $\boldsymbol{k}$ ), and $\alpha$ is an angle chosen for the orientation of the spectral wavevector $\hat{\boldsymbol{u}}$. By imposing the ratio of poloidal to toroidal energy $\mathcal{E}^{(2)}\left(k, O_{6}\right) / \mathcal{E}^{(1)}\left(k, O_{6}\right)$, one also imposes the wavevector orientation and thus the ratio between the poloidal and toroidal components $u^{(2)}$ and $u^{(1)}$. Geometrically, there are four possibilities for the angle $\alpha$ (red arrows plotted in Figure 9), so that one out of four is randomly chosen for each wavevector involved in the sum in Eq. (11). We plot in Figure 9 the vorticity field and 300 random streamlines in our synthetic model of polarized velocity field for two ratios:

- $\mathcal{E}^{(2)}\left(k, O_{6}\right) / \mathcal{E}^{(1)}\left(k, O_{6}\right)=1 / 3$, so that $\beta \in\left\{\frac{\pi}{6},-\frac{\pi}{6}, \frac{5 \pi}{6},-\frac{5 \pi}{6}\right\}$ : the horizontal velocity component is more important than the vertical one.

- $\mathcal{E}^{(2)}\left(k, O_{6}\right) / \mathcal{E}^{(1)}\left(k, O_{6}\right)=3$, so that $\beta \in\left\{\frac{\pi}{3},-\frac{\pi}{3}, \frac{2 \pi}{3},-\frac{2 \pi}{3}\right\}$ : the horizontal velocity component is less important than the vertical one.

Comparing Figures 9(a) and 9(c) for the vorticity surfaces, it follows that the vorticity fields exhibit very similar features in both cases, with the same amplitude and same vertically oriented structures. But by comparing Figures 9(b) and 9(d), one is able to tell one case from the other, since the streamlines are clearly different: for the poloidally polarized case $\mathcal{E}^{(2)}\left(k, O_{6}\right) / \mathcal{E}^{(1)}\left(k, O_{6}\right)=3$ of Figure 9(d), the streamlines are loosely helical and strongly aligned with the vertical direction ("jetal" eddies); whereas for the toroidally polarized case $\mathcal{E}^{(2)}\left(k, O_{6}\right) / \mathcal{E}^{(1)}\left(k, O_{6}\right)=1 / 3$, the streamlines swirl more horizontally ("vortical” eddies). 

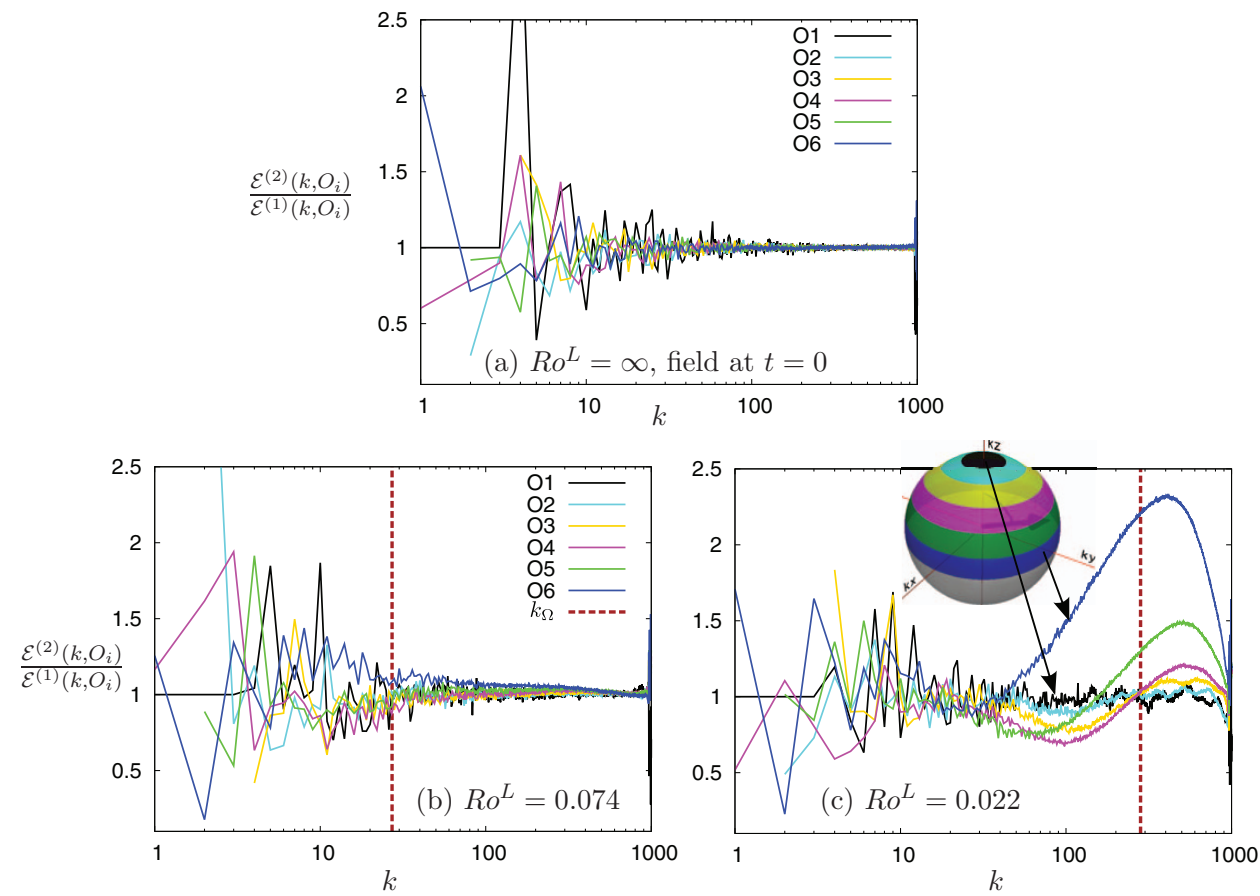

FIG. 10. Ratio of poloidal to toroidal energy $\mathcal{E}^{(2)}\left(k, O_{i}\right) / \mathcal{E}^{(1)}\left(k, O_{i}\right)$ for different angular sectors at time $t=t_{f}$ and for different Rossby numbers $R o^{L}$ at fixed resolution $2048^{3}$ : (a) Isotropic initialisation $\left(R o^{L}=\infty\right)$; (b) Run $B_{2}, R o^{L}=0.074$; and (c) Run $B_{1}, R o^{L}=0.022$.

\section{Polarization in DNS fields}

We plot in Figure 10 the ratio $\mathcal{E}^{(2)}\left(k, O_{i}\right) / \mathcal{E}^{(1)}\left(k, O_{i}\right)$ for different angular sectors (rings) in the isotropic case (initial condition) and for two Rossby numbers $R o^{L}=0.074$ and $R o^{L}=0.022$ at $t=t_{f}$. The ratio $\mathcal{E}^{(2)}\left(k, O_{i}\right) / \mathcal{E}^{(1)}\left(k, O_{i}\right)$ depends on both the angle (ring) and on the scale (spectral shell). In isotropic turbulence in Figure $10\left(\right.$ a), we observe that $\mathcal{E}^{(2)}\left(k, O_{i}\right) / \mathcal{E}^{(1)}\left(k, O_{i}\right)$ collapses to unity for all the rings, so that the toroidal and poloidal velocity components $u^{(1)}$ and $u^{(2)}$ have the same magnitude on average. In addition, one recovers that all rings have the same contribution to energy, and that the two components of velocity are statistically equivalent in each ring. Overall, one concludes that the polarization and directional anisotropies are zero, as expected. Not only isotropic turbulence permits to test this, but the curves in Figure 10(a) provide an estimate of the wiggles expected in the plots due to limited statistical sampling, with very few spectral points contained in the largest scales.

In contrast with isotropic turbulence, for the rotating case at $R o^{L}=0.074$ presented in Figure 10(b), the magnitude of the poloidal/toroidal energy ratio is significantly different from unity, especially for the equatorial ring $O_{6}$ : poloidal energy is larger than toroidal energy in the large scales at wavenumbers smaller than the Zeman scale. The anisotropy at these scales is reversed when considering the rings farther from the equator. When considering smaller scales, the anisotropy monotonically decreases in all the rings, with values matching unity only in the smallest scales. We therefore note that, in the small scales corresponding to wavenumbers above the Zeman scale, where turbulence is supposed to be isotropic from the point of view of directional anisotropy, there still remains a hint of polarization anisotropy. This is not due to a numerical artifact, from comparison with the isotropic case of Figure 10(a). 
We now consider the lower Rossby number case $R o^{L}=0.022$ of Figure $10(\mathrm{c})$, for which the behavior of polarization anisotropy is more complex:

- Looking at the equatorial ring $O_{6}$, the toroidal component weakly prevails at the largest scales $\left(\mathcal{E}^{(2)}\left(k, O_{6}\right)<\mathcal{E}^{(1)}\left(k, O_{6}\right)\right)$ - although this is somehow drowned in the sampling oscillations - whereas the reverse tendency $\mathcal{E}^{(2)}\left(k, O_{6}\right)>\mathcal{E}^{(1)}\left(k, O_{6}\right)$ appears at smaller scales $k>42$. From this scale up to $k=411$ we observe a strong increase of the polarization anisotropy ratio, ending with a peak $\mathcal{E}^{(2)}\left(k, O_{6}\right)=2.33 \mathcal{E}^{(1)}\left(k, O_{6}\right)$. After this peak, the ratio quickly decreases back to unity in the dissipative range.

- The other rings $\mathrm{O}_{5}, \mathrm{O}_{4}, \mathrm{O}_{3}, \mathrm{O}_{2}$ show similar variations, although with less magnitude for each successive ring when approaching the polar direction. We note that, for these rings and especially the three closer to equator, the Zeman scale $k_{\Omega}$ is close to the peak of the poloidally dominated zone. Below, the further the $k$ from $k_{\Omega}$ the smaller the ratio $\mathcal{E}^{(2)}\left(k, O_{i}\right) / \mathcal{E}^{(1)}\left(k, O_{i}\right)$, and similarly above. (One could also pretend to observe a similar effect on ring $\mathrm{O}_{6}$ of Figure 10(b).) This spectacular effect of the Zeman scale could be fortuitous but it carries an additional significance at low Rossby number $R o^{L}$ : the excess of toroidal component $\mathcal{E}^{(1)}\left(k, O_{i}\right)$ at larger scale is progressively balanced by an increasing excess of poloidal component $\mathcal{E}^{(2)}\left(k, O_{i}\right)$ when moving to smaller scales, until the smallest scale isotropy is restored such that $\mathcal{E}^{(2)}\left(k, O_{i}\right) \simeq \mathcal{E}^{(1)}\left(k, O_{i}\right)$. (There is of course not global balance over all scales.) The non-monotonous behavior of the spectra is the result of a competition of linear and nonlinear terms for polarization at $k_{\|} \neq 0$ (see Eq. (A2)).

- Finally, the ring $O_{1}$ has a statistically isotropic contents $\mathcal{E}^{(2)}\left(k, O_{1}\right) \simeq \mathcal{E}^{(1)}\left(k, O_{1}\right)$ with a mostly unit poloidal/toroidal ratio. This is consistent with axisymmetry and is sometimes denoted "polar isotropy."

The energy at the equator and the difference between $\mathcal{E}^{(1)}\left(k, O_{i}\right)$ and $\mathcal{E}^{(2)}\left(k, O_{i}\right)$ at all scale is a strong characteristic of the quasi-2D-3C state. From the rings $O_{6}$ of Figures $10(\mathrm{~b})$ and $10(\mathrm{c})$, we confirm that the large-scale structures of the flow are anisotropic, with dominance of jetal eddies along the rotation axis. In the larger Rossby number case of Figure 10(b), smaller scales do not exhibit significant polarization anisotropy, hence the classical-turbulence-like small vortices observed in Figure 6(c), which are superimposed on large-scale axially oriented clusters. However, in Figure 10(c) the polarization anisotropy is strong at small scales, so that one expects small-scale structures axially oriented, a feature which is confirmed when observing closely the $3 \mathrm{D}$ visualization of Figure 6(d). Moreover, the strong equatorial polarization is linked to small-scale structures that are similar to the ones observed in our strongly polarized model of Sec. III D 1 and plotted in Figure 9. Finally, within the corresponding small-scale range, from the analysis of both equatorial spectra and poloidal mode dominance, the energy of the flow in the axial direction is larger than in the horizontal direction, so that velocity components are statistically ordered such that $u_{\|}>u_{\perp}$.

\section{E. Anisotropy reflected by the spectral energy transfer}

After investigating the structure of rotating turbulence via spectra of two-point velocity correlations and the related directional and polarization anisotropies, we hereafter propose to consider the third-order moments corresponding to the nonlinear energy transfer terms. Our goal is to try to identify the mechanisms that create the anisotropy. From Eq. (4) we deduce the equation of energy mode by mode, at given $\boldsymbol{k}$ :

$$
\left(\frac{\partial}{\partial t}+2 v k^{2}\right) e(\boldsymbol{k}, t)=s(\boldsymbol{k}, t)
$$

where $e(\boldsymbol{k}, t)=|\hat{\boldsymbol{u}}(\boldsymbol{k}, t)|^{2} / 2$ is the (unaveraged) energy for mode $\boldsymbol{k}$ and the nonlinear energy transfer term is

$$
s(\boldsymbol{k}, t)=-\Re\left[\hat{\boldsymbol{u}}^{*}(\boldsymbol{k}) \cdot \boldsymbol{P}(\boldsymbol{k})\left(\sum_{p+q=k} \hat{\boldsymbol{\omega}}(\boldsymbol{p}) \times \hat{\boldsymbol{u}}(\boldsymbol{q})\right)\right],
$$



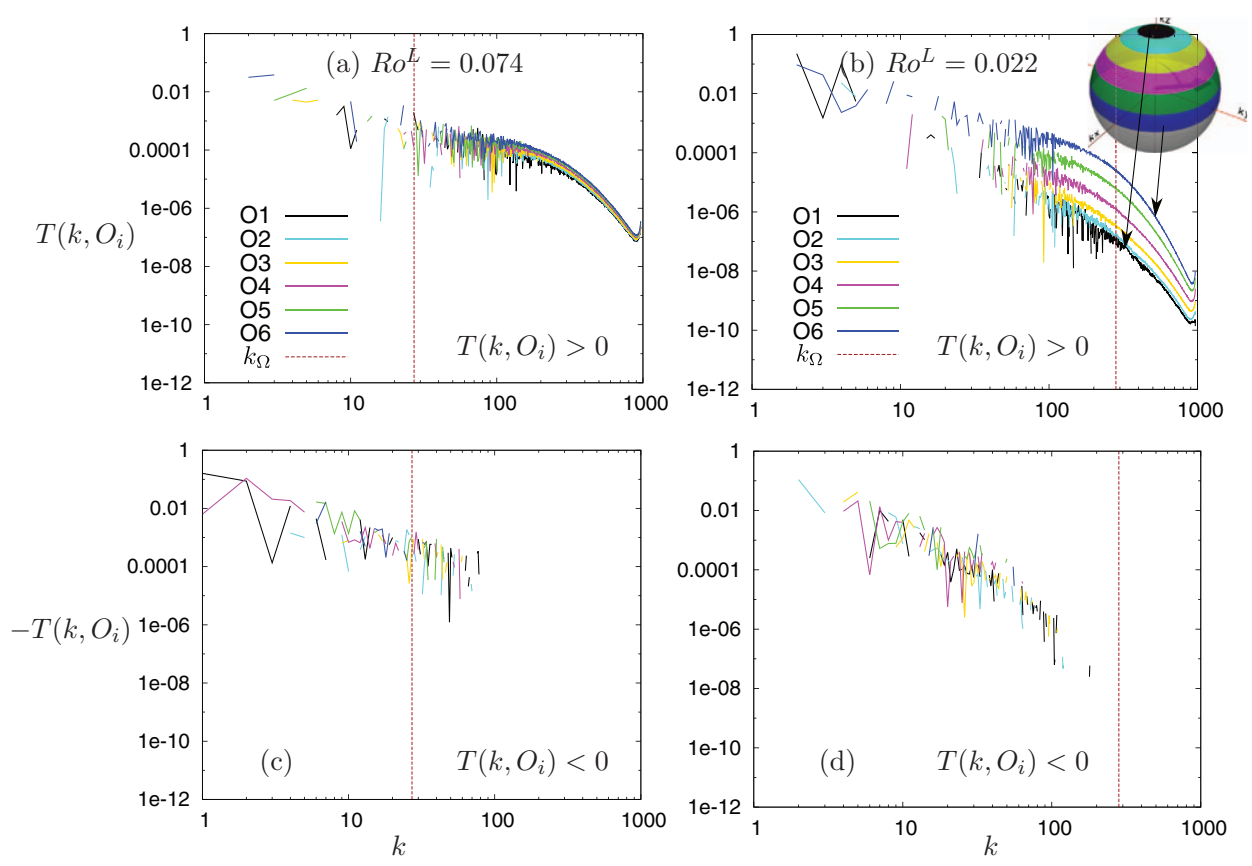

FIG. 11. Logarithmic plot of the transfer of spectral kinetic energy $T\left(k, O_{i}\right)$ at $t=t_{f}$ for different Rossby numbers $R o^{L}$ at fixed resolution $2048^{3}$ : positive part of the transfer (a) for run $A_{5}, R o^{L}=0.074$ and (b) for run $A_{6}, R o^{L}=0.022$. Negative part of the transfer (c) for run $A_{5}, R o^{L}=0.074$ and (d) for run $A_{6}, R o^{L}=0.022$.

from the discretized form of Eq. (4) or (6), in which the projection operator is defined in Eq. (5). Note that rotation does not appear explicitly in the energy equation (12) but implicitly in the velocity $\boldsymbol{u}(\boldsymbol{k}, t)$ through nonlinearity and anisotropic pressure re-distribution.

The Lin equation for the kinetic energy spectrum is obtained by averaging Eq. (12) for $e(\boldsymbol{k}, t)$ over the surface of a sphere $S_{k}$, i.e., $E(k, t)=\sum_{k \in S_{k}} e(\boldsymbol{k}, t)$ and the nonlinear energy transfer is given scale by scale by $T(k, t)=\sum_{k \in S_{k}} s(\boldsymbol{k}, t)$. In the case of freely rotating turbulence, this nonlinear energy transfer remains negative at large scale and positive at small scale, and thus corresponds to a direct cascade of energy, as in isotropic turbulence, but its amplitude is decreased by rotation. ${ }^{16,20}$

As before, in order to characterize the anisotropy, we introduce the angular dependence of the nonlinear energy transfer $T^{(e)}\left(k, O_{i}\right)$ which is defined as the angle-dependent transfer ${ }^{20}$ related to the angle-dependent spectral energy $\mathcal{E}\left(k, O_{i}\right)$ defined in (9):

$$
T^{(e)}\left(k, O_{i}\right)=\sum_{k \in O_{i}} s(\boldsymbol{k}, t)
$$

We plot $T^{(e)}\left(k, O_{i}\right)$ at final time $t=t_{f}$ in Figure 11. Since $T^{(e)}\left(k, O_{i}\right)$ is a third-order statistics, its computation in practice is more demanding than second-order statistics such as energy spectra. Accordingly, only the high resolution case $\left(2048^{3}\right)$ appears on this figure because the results are very noisy: a large number of Fourier modes are needed for each ring, in the absence of time averaging not possible in freely decaying flows. We observe in both cases that the nonlinear energy transfer $T^{(e)}\left(k, O_{i}\right)$ is mostly negative at large scale (Figures $11(\mathrm{c})$ and $11(\mathrm{~d})$ ) and positive at small scale (Figures 11(a) and 11(b)) for each ring, attesting of dominant downscale cascade of energy.

At the larger Rossby number case of Figures 11(a) and 11(c), the nonlinear transfer contribution to every ring $T^{(e)}\left(k, O_{i}\right)$ is almost equivalent, for every scale and every angle, especially at the smallest scales, as a trace of close-to-isotropic transfer. The transfer at the equatorial ring $O_{6}$ is nonetheless 
slightly dominant. At large scales, lack of sampling prevents a definitive observation. In contrast, at lower Rossby number in Figures 11(b) and 11(d), the nonlinear energy transfer concentrates in the equatorial ring $O_{6}$. This result is consistent with direct measurements of two-point anisotropic third-order structure function, ${ }^{3,10}$ which carries the same information as the angle-dependent spectral energy transfer does.

Consequently, we directly show that the anisotropic distribution of $\mathcal{E}\left(k, O_{1}\right)$ is driven by the anisotropic structure of the nonlinear transfer term. The difference between $\mathcal{E}^{(1)}\left(k, O_{1}\right)$ and $\mathcal{E}^{(2)}\left(k, O_{1}\right)$ has the same origin, even if the linear effect of phase-mixing by inertial waves tends to damp the polarization anisotropy. ${ }^{20,23}$ In addition, the Zeman wavenumber $k_{\Omega}$ is shown to delineate the return to isotropy of the nonlinear energy transfer, since all contributions $T^{(e)}\left(k, O_{i}\right)$ tend to gather if $k>k_{\Omega}$, for every ring.

\section{CONCLUSION}

We have shown that the Zeman wavenumber provides a reliable threshold for restoring isotropy within the inertial range, using high resolution DNS of decaying rotating turbulence. This is first demonstrated on the angle-dependent distribution of spectral energy, which characterizes directional anisotropy.

In conventional anisotropic turbulence, in which energy and anisotropy are created at large scale either by a production term (as in sheared turbulence) or by forcing, the anisotropy is monotonically decaying from the smallest $k$ to the largest one, and it is not surprising to have a threshold wavenumber above which anisotropy is no longer significant. This conventional behavior is not inconsistent with the analysis of Zhou et al. ${ }^{27}$ who show that in the case of anisotropic forcing at large scale the transfer between small scales depend mainly on this large scale anisotropy. An important question to be addressed is therefore to which extent re-isotropization of smallest scales means restoration of universality. Do we have evidence of a clear change of slope, with restoration of a $-5 / 3$ slope? We provide a partial answer in this work.

The main contrast with conventional anisotropic turbulence is the fact that anisotropy can affect a range of small scales, so that anisotropy can be increasing from smallest wavenumbers to largest wavenumbers if $k_{\Omega}$ is very large. This result is consistent throughout all previous numerical ${ }^{1}$ and theoretical studies, using DNS, inertial wave-turbulence theory, ${ }^{19}$ and anisotropic eddy-damped quasi-normal Markovian (EDQNM) two-point models. ${ }^{20}$ This anisotropy at small scales was recently confirmed by direct measurements of anisotropic energy transfer in the Gyroflow experiment. ${ }^{10}$ This suggested that, if isotropy is restored for $k>k_{\Omega}$, it does not decrease monotonically from the smallest $k^{\prime} \mathrm{s}$ to $k_{\Omega}$ but reaches a maximum at a given wavenumber $k_{\text {max }}^{\text {aniso }}$ significantly smaller than $k_{\Omega}$. This behavior is demonstrated here, for the first time using very high resolution DNS. For this purpose, the fully axisymmetric angle-dependent energy spectrum has been extracted from DNS, using both a shell-to-shell and a ring-to-ring description. Directional anisotropy consists of an accumulation of energy, at a given $k$ (shell), from the polar ring to the equatorial ring, in a monotonic way. This anisotropy, consistent with a saturated trend from 3D to 2D structure, seems to increase from the smallest $k$ to a maximum $k_{\max }^{\text {aniso }}$, and then clearly decreases from $k_{\max }^{\text {aniso }}$ to wavenumbers slightly larger than the Zeman wavenumber $k_{\Omega}$, before possible re-isotropization for higher $k$ s.

In addition, polarization anisotropy has been explored, distinguishing the contributions from toroidal — zonal in the polar-spherical system of coordinates on the sphere - and poloidal meridional in the same system - velocity components. The role of the Zeman wavenumber is similar to its role played in directional anisotropy, but the maximum of polarization, located at the equatorial ring, is found at a wavenumber larger than $k_{\Omega}$, even if polarization decreases at even higher wavenumbers. In addition, the polarization can be inversed between the range $\left(k_{\min }, \sim k_{\Omega}\right)$ and $\left(\sim k_{\Omega}, k_{\max }\right.$ ) for rings other than the equatorial and the polar ones.

Finally, the results on directional anisotropy for the angle-dependent energy spectrum are recovered from the nonlinear energy transfer. Since the Coriolis force produces no energy, there is no explicit production term in the energy equation, and the anisotropic distribution of energy is closely related to the nonlinear energy transfer mediated by cubic correlations (in the right-handsides of the statistically averaged equation (12)). The crucial role and related anisotropic distribution 
of the third-order structure function, which is the counterpart of the anisotropic nonlinear spectral transfer, was shown experimentally by Lamriben et al. (2011), ${ }^{10}$ in agreement with our numerical results.

\section{ACKNOWLEDGMENTS}

We thank Y. Kaneda's group for providing the isotropic turbulence fields we used for the $2048^{3}$ DNS initialization. Our simulations were performed on the IDRIS computing center of CNRS, thanks to cpu time allocation provided by GENCI under Project No. 22206.

\section{APPENDIX: DYNAMICAL EQUATIONS FOR THE POLARIZATION ANISOTROPY}

We recall that the velocity can be split in toroidal and poloidal contributions $u^{(1)}$ and $u^{(2)}$ from Eq. (7), and that the corresponding spectra $\mathcal{E}^{(1)}$ and $\mathcal{E}^{(2)}$ add up to the axisymmetric kinetic energy spectrum

$$
\mathcal{E}(\boldsymbol{k})=\mathcal{E}^{(1)}(\boldsymbol{k})+\mathcal{E}^{(2)}(\boldsymbol{k}),
$$

whose dynamics is described by Lin's equation. Separate equations can be obtained for the toroidal and poloidal spectra (details in the book by Sagaut and Cambon ${ }^{11}$ ). One needs to introduce also the complex polarization spectrum $Z(\boldsymbol{k}, t)$, whose real part is $\Re Z=\mathcal{E}^{(2)}-\mathcal{E}^{(1)}$ and imaginary part is the toroidal-poloidal cross spectrum $\mathfrak{I} Z=\mathfrak{J}\left(\left\langle u^{(1)}(\boldsymbol{k}, t) u^{(2)}(\boldsymbol{k}, t)\right\rangle\right)$. (The real part $\Re\left(\left\langle u^{(1)}(\boldsymbol{k}, t) u^{(2)}(\boldsymbol{k}, t)\right\rangle\right)$ is related to helicity, absent in our simulations since initially zero.)

Separate Lin-type equations for $\mathcal{E}(\boldsymbol{k}, t)$ and $Z(\boldsymbol{k}, t)$ are obtained from the two-point velocity correlation in Fourier space, as

$$
\begin{gathered}
\left(\frac{\partial}{\partial t}+2 v k^{2}\right) \mathcal{E}(\boldsymbol{k}, t)=T^{(e)}(\boldsymbol{k}, t), \\
\left(\frac{\partial}{\partial t}+2 v k^{2}+4 \mathrm{i} \boldsymbol{\Omega} \cdot \frac{\boldsymbol{k}}{k}\right) Z(\boldsymbol{k}, t)=T^{(z)}(\boldsymbol{k}, t),
\end{gathered}
$$

with $T^{(e)}(\boldsymbol{k}, t)$ the kinetic energy nonlinear transfer, and $T^{(z)}(\boldsymbol{k}, t)$ the polarization nonlinear transfer. Note the rotating-phase term in (A2), proportional to rotation and to the wavevector orientation. This term vanishes at $k_{\|}=0$ so that dominant polarization is governed by $T^{(z)}$. The half-sum and halfdifference between Eq. (A1) and real part of (A2) provide the dynamical equations for the poloidal spectrum $\mathcal{E}^{(2)}$ and toroidal one $\mathcal{E}^{(1)}$.

${ }^{1}$ C. Cambon, N. N. Mansour, and F. S. Godeferd, "Energy transfer in rotating turbulence,” J. Fluid Mech. 337, 303-332 (1997).

${ }^{2}$ P. A. Davidson, P. J. Staplehurst, and S. B. Dalziel, “On the evolution of eddies in a rapidly rotating system," J. Fluid Mech. 557, 135-144 (2006)

${ }^{3}$ P.-P. Cortet and F. Moisy, "Decaying turbulence in a rotating frame: Which scales are more anisotropic?," J. Fluid Mech. (submitted).

${ }^{4}$ O. Zeman, "A note on the spectra and decay of rotating homogeneous turbulence," Phys. Fluids 6, 3221 (1994).

${ }^{5}$ S. Corrsin, "On local isotropy in turbulent shear flow," Technical Report 58B11, NACA R \& M, 1958.

${ }^{6}$ T. M. Dillon, "Vertical overturns: A comparison of Thorpe and Ozmidov length scales," J. Geophys. Res. 87(C12), 9601-9613, doi:10.1029/JC087iC12p09601 (1982).

${ }^{7}$ H. P. Greenspan, The Theory of Rotating Fluids (Cambridge University Press, 1968).

${ }^{8}$ P. D. Mininni, D. Rosenberg, and A. Pouquet, "Isotropisation at small scales of rotating helically driven turbulence," J. Fluid Mech. 699, 263-279 (2012).

${ }^{9}$ L. Jacquin, O. Leuchter, C. Cambon, and J. Mathieu, "Homogeneous turbulence in the presence of rotation,” J. Fluid Mech. 220, 1-52 (1990).

${ }^{10}$ C. Lamriben, P.-P. Cortet, and F. Moisy, "Direct measurements of anisotropic energy transfers in a rotating turbulence experiment," Phys. Rev. Lett. 107(2), 024503 (2011).

${ }^{11}$ P. Sagaut and C. Cambon, Homogeneous Turbulence Dynamics (Cambridge University Press, 2008).

${ }^{12}$ D. Pekurovsky, "P3DFFT: A framework for parallel computations of fourier transforms in three dimensions," SIAM J. Sci. Comput. 34(4), C192-C209 (2012).

${ }^{13}$ Y. Hussaini, A. Quarteroni, and C. G. Canuto, Spectral Methods: Evolution to Complex Geometries and Applications to Fluid Dynamics, Scientific Computation (Springer, London, 2007). 
${ }^{14}$ T. Aoyama, T. Ishihara, Y. Kaneda, M. Yokokawa, K. Itakura, and A. Uno, "Statistics of energy transfer in high-resolution direct numerical simulation of turbulence in a periodic box," J. Phys. Soc. Japan 74(12), 3202-3212 (2005).

${ }^{15}$ J. R. Herring, “Approach of axisymmetric turbulence to isotropy,” Phys. Fluids 17(5), 859-872 (1974).

${ }^{16}$ Y. Morinishi, K. Nakabayashi, and S. Q. Ren, "Dynamics of anisotropy on decaying homogeneous turbulence subjected to system rotation,” Phys. Fluids 13(10), 2912-2922 (2001).

${ }^{17}$ C. Morize, F. Moisy, and M. Rabaud, “Decaying grid-generated turbulence in a rotating tank," Phys. Fluids 17(9), 095105 (2005).

${ }^{18}$ Y. Zhou, "A phenomenological treatment of rotating turbulence," Phys. Fluids 7, 2092 (1995).

${ }^{19}$ F. Bellet, F. S. Godeferd, J. F. Scott, and C. Cambon, "Wave turbulence in rapidly rotating flows," J. Fluid Mech. 562, 83-121 (2006).

${ }^{20}$ C. Cambon and L. Jacquin, "Spectral approach to non-isotropic turbulence subjected to rotation," J. Fluid Mech. 202, 295-317 (1989).

${ }^{21}$ F. S. Godeferd and C. Staquet, "Statistical modelling and direct numerical simulations of decaying stably-stratified turbulence: Part 2: Large scales and small scales anisotropy,” J. Fluid Mech. 486, 115-150 (2003).

${ }^{22}$ F. Moisy, C. Morize, M. Rabaud, and J. Sommeria, "Decay laws, anisotropy and cyclone-anticyclone anisotropy in decaying rotating turbulence," J. Fluid Mech. 666, 5-35 (2011).

${ }^{23}$ C. Cambon, L. Danaila, F. S. Godeferd, and J. F. Scott, "Third-order statistics and the dynamics of strongly anisotropic turbulent flows," J. Turbul. 14(3), 121-160 (2013)

${ }^{24}$ J. Proudman, "On the motion of solids in a liquid possessing vorticity," Proc. R. Soc. London 92(642), 408-424 (1916).

${ }^{25}$ G. I. Taylor, "Motion of solids in fluids when the flow is not irrotational," Proc. R. Soc. London 93(648), 99-113 (1917).

${ }^{26}$ R. S. Rogallo, "Numerical experiments in homogeneous turbulence," Technical Report 81315, NASA, 1981.

${ }^{27}$ Ye Zhou, P. K. Yeung, and James G. Brasseur, "Scale disparity and spectral transfer in anisotropic numerical turbulence," Phys. Rev. E 53(1), 1261-1264 (1996). 